ORIGINAL ARTICLE

\title{
On issues of confidence in determining the time constant for oxygen uptake kinetics
}

\author{
G H Markovitz, J W Sayre, T W Storer, C B Cooper
}

Br J Sports Med 2004;38:553-560. doi: 10.1136/bjsm.2003.004721

See end of article for authors' affiliations

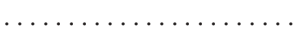

Correspondence to: Dr Cooper, 37-131 CHS UCLA Medical Center, 10833 Le Conte Avenue, Los Angeles, CA 90095,

USA; ccooper@mednet. ucla.edu

Accepted 29 July 2003
Background: $\tau \dot{V} \mathrm{O}_{2}$ at the onset of constant work rate (CWR) exercise is a variable of aerobic fitness that shortens with physical training and lengthens with cardiopulmonary disease. Determination of $\tau \mathrm{V}_{2}$ with sufficiently high confidence has typically required multiple exercise transitions limiting its clinical application.

Objectives: To design a protocol to determine $\tau \dot{\mathrm{V}} \mathrm{O}_{2}$ reliably but simply.

Methods: On each of three days, five healthy men performed two CWR tests on a cycle ergometer below the metabolic threshold $\left(\dot{V}_{2} \theta\right)$ for blood lactate accumulation as determined by gas exchange measurements followed by an incremental work rate (IWR) test. $\tau \dot{\mathrm{V}} \mathrm{O}_{2}$ was determined (a) from the ontransit (on- $\tau \dot{\mathrm{V}} \mathrm{O}_{2}$ ) and off-transit (off- $\tau \dot{\mathrm{V}} \mathrm{O}_{2}$ ) of six CWR tests both individually and superimposed, using non-linear regression with a monoexponential model, and (b) by geometric analysis of the IWR tests (ramp- $\tau \dot{\mathrm{V}} \mathrm{O}_{2}$ ).

Results: Group means (SD) were: $\dot{V} \mathrm{O}_{2} \mathrm{MAX} 3.84$ (0.44) litres/min, $\dot{\mathrm{V}} \mathrm{O}_{2} \theta 1.88$ (0.23) litres/min, steady state exercise $\dot{\mathrm{V}}_{2} 1.67$ (0.07) litres/min, on- $\tau \dot{\mathrm{V}} \mathrm{O}_{2} 38.0$ (5.3) seconds, off- $\tau \dot{\mathrm{V}} \mathrm{O}_{2} 39.0$ (4.3) seconds, and ramp- $\tau \dot{\mathrm{V}} \mathrm{O}_{2} 60.8$ (15.4) seconds. On- $\tau \dot{\mathrm{V}} \mathrm{O}_{2}$ correlated with off- $\tau \dot{\mathrm{V}} \mathrm{O}_{2}(r=0.87), \dot{\mathrm{V}} \mathrm{O}_{2} \mathrm{MAX}(r=-0.73)$, and $\dot{\mathrm{V}} \mathrm{O}_{2} \theta(r=0.89)$. The pooled mean $\tau \dot{\mathrm{V}} \mathrm{O}_{2}$ from six superimposed tests agreed with the arithmetic grand mean of the six tests.

Conclusions: The average of on- $\tau \dot{\mathrm{V}} \mathrm{O}_{2}$ and off- $\tau \dot{\mathrm{V}} \mathrm{O}_{2}$ fell within the $95 \%$ confidence interval of the pooled mean by the second test. Ramp- $\tau \dot{V} \mathrm{O}_{2}$ was longer and less reproducible. These findings support the use of both on- and off-transit data for the determination of $\tau \dot{\mathrm{V}} \mathrm{O}_{2}$, an approach that reduces the number of transitions necessary for accurate determination of $\tau \dot{\mathrm{V}} \mathrm{O}_{2}$, potentially enhancing its clinical application.
$\mathrm{T}$ he ability to exercise is an integral component of physical and psychological well being. The exercise test is widely used to study physical fitness and assess the effects of exercise training. In addition, exercise testing may be used to evaluate cardiopulmonary or neuromuscular disease and the response to rehabilitation. The ability to sustain high intensity exercise depends on four aerobic variables: $(a)$ the maximum oxygen uptake $\left(\dot{\mathrm{V}}_{2} \mathrm{MAX}\right),(b)$ the metabolic threshold $\left(\dot{\mathrm{V}}_{2} \theta\right)$ above which there is a sustained increase in blood lactate, $(c)$ the work efficiency $(\eta)$, and $(d)$ the time constant for oxygen uptake $\left(\tau \dot{\mathrm{V}}_{2}\right){ }^{1}$

The time constant for oxygen uptake kinetics was first described by Margaria et al in 1933 . $^{2}$ With an increase in activity or exercise, there is a transient non-steady-state period during which physiological adaptations adjust to meet the increased metabolic demand. The rate of change in $\dot{\mathrm{V}}_{2}$ becomes proportionally smaller as the subject approaches a new steady state. This relation can be characterised by first order kinetics. ${ }^{3}{ }^{4}$ The time constant mathematically describes the profile of this adaptive phase and is a reflection of the response of the cardiovascular system and muscles to a step up in external work rate. The wash-in exponential function that describes this relation and therefore calculates instantaneous $\dot{\mathrm{V}}_{2 \mathrm{t}}$ at time $\mathrm{t}$ is as follows:

$$
\dot{\mathrm{V}}_{2 \mathrm{t}}=\Delta \tau \dot{\mathrm{V}}_{2}\left(1-\mathrm{e}^{(-\mathrm{t} / \tau)}\right)
$$

where $\Delta$ is the increase in steady state $\dot{\mathrm{V}}_{2}$ and $\tau$ is the time constant (see fig 1 , equation 3 ).

As one of the four key variables of aerobic function, $\tau \dot{\mathrm{V}}_{2}$ should be a useful clinical or research measure in the assessment of cardiovascular and pulmonary disease, fitness, and the effects of exercise training. Previous studies have reported $\tau \dot{\mathrm{V}}_{2}$ values ranging from 35 seconds $^{5}$ up to 50 seconds $^{1}$ in normal subjects, 25 seconds in highly trained athletes, ${ }^{6}$ and $63-75$ seconds in patients with diagnosed cardiopulmonary disease. ${ }^{78}$ Endurance training has been shown to accelerate $\dot{\mathrm{V}}_{2}$ kinetics in previously untrained healthy subjects by the fourth day of exercise. ${ }^{8}$

Usually a clinical exercise test reports $\dot{\mathrm{V}}_{2} \mathrm{MAX}$ and $\dot{\mathrm{V}}_{2} \theta$, substitutes the oxygen uptake/work rate relation $\left(\Delta \dot{\mathrm{V}}_{2} / \Delta \dot{\mathrm{W}}\right)$ for work efficiency, but rarely derives the time constant. The commonly practiced methodology to determine $\tau \dot{\mathrm{V}} \mathrm{O}_{2}$ involves four to eight constant work rate (CWR) exercise tests. ${ }^{34-12}$ The variability of breath by breath data particularly at rest led to the necessity for multiple tests. This practice is tedious and time consuming, rendering it impractical for clinical use. A less arduous protocol would be desirable for clinical purposes. A single incremental work rate (IWR) test, also referred to as a ramp test, has been advocated to determine $\tau \dot{\mathrm{V}}_{2}$ as well as the other variables of aerobic function. Comparable results between IWR and a single CWR test have been reported. ${ }^{1} \mathrm{~A}$ variety of ramp slopes $(20,30$, and $50 \mathrm{~W} / \mathrm{min})$ may be used with equal reliability. ${ }^{13}$

Both the single ramp test and the CWR test are far simpler and less time consuming than the standard multiple CWR exercise tests. However, neither approach has been rigorously evaluated against the "gold standard" of performing multiple CWR tests. Therefore, we proposed that $\tau \dot{\mathrm{V}}_{2}$ is a reproducible measure of aerobic performance, that the reproducibility of $\tau \dot{\mathrm{V}} \mathrm{O}_{2}$ may be improved with a protocol that maximises the signal/noise ratio, that $\tau \dot{\mathrm{V}}_{2}$ is similarly exhibited at the onset and offset of exercise, and that $\tau \dot{\mathrm{V}}_{\mathrm{O}_{2}}$ may be determined with equal validity from an IWR or a CWR protocol.

Abbreviations: CWR, constant work rate; IWR, incremental work rate 


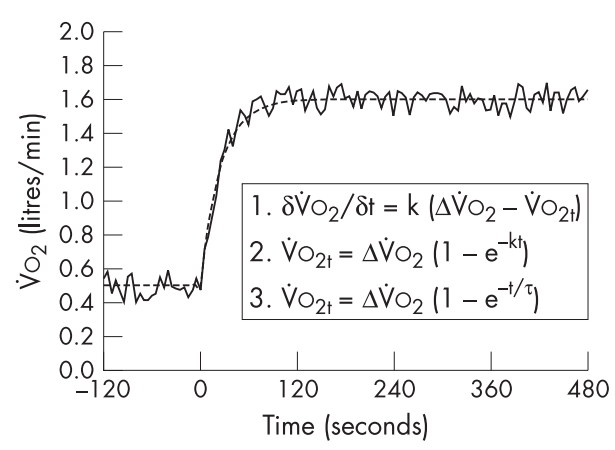

Figure 1 Monoexponential increase in $\dot{\mathrm{V}}_{2}$ in response to constant work rate exercise below the metabolic threshold for blood lactate accumulation. Equation 1 illustrates that the rate of change in $\mathrm{V}_{2}$ becomes proportionally smaller $(\mathrm{k})$ as the subject approaches the new steady state. Equation 2 derives the instantaneous $\mathrm{VO}_{2}$ at time $+\left(\mathrm{V}_{2 t}\right)$. Equation 3 introduces the time constant $(\tau)$ where $\tau=1 / k$.

\section{METHODS}

\section{Subjects}

Ten healthy male subjects, aged 21-33 years, volunteered to take part in this study and gave their informed consent. Five were subsequently excluded from the analysis for reasons explained below related to $\dot{\mathrm{V}}_{2}$ drift. All were participants in some form of recreational exercise, but none were physically trained, which we defined as performing more than moderate intensity exercise for more than 30 minutes three times a week. The subjects were recruited from the local and university community, and were non-smokers specifically free from cardiopulmonary and neuromuscular disease. All subjects signed a consent form approved by the UCLA Human Subjects in Research Protection Committee (IRB).

\section{Experimental design}

To evaluate the variables of aerobic fitness, each subject performed a series of exercise tests on an electromagnetically braked cycle ergometer in the upright position. We calibrated the cycle ergometer before this study using a running torque cycloergometer calibrator (model 17801; Vacumed, Ventura, California, USA). The protocol consisted of two CWR tests followed by one IWR test each session. We allowed a rest period of 30 minutes between the CWR tests and the IWR test. We considered this interval sufficient because the CWR tests were intentionally below the threshold for lactate accumulation. The protocol took place on three nonconsecutive days for a total of six CWR tests and three IWR tests. The subjects did not vary the level of their usual exercise activity during the testing period.

We designed a CWR protocol that would optimise the signal/noise ratio while attempting to remain below the metabolic threshold $\left(\dot{\mathrm{V}}_{2} \theta\right)$ above which blood lactate accumulates causing a disproportionate rise in minute ventilation $(\dot{\mathrm{V}} \mathrm{E})$ and carbon dioxide output $\left(\dot{\mathrm{V}}_{\mathrm{CO}_{2}}\right)$ relative to $\dot{\mathrm{V}}_{2}$. Previous experience with this type of subject in our laboratory led us to choose the following regimen: four minute warm up phase at $20 \mathrm{~W}$ (baseline), followed by an abrupt transition to the exercise phase at $120 \mathrm{~W}$ sustained for eight minutes (steady state), and then an abrupt transition to an eight minute recovery period at $20 \mathrm{~W}$ (recovery). Each test was performed on an electromagnetically braked cycle ergometer (Ergoline $800 \mathrm{~S}$ ) with the subjects wearing a nose clip and breathing through a mouthpiece connected to a low resistance valve. The subjects were asked to maintain a cycling cadence of $60 \mathrm{rpm}$ throughout all phases of each CWR test.

After the two CWR tests, each subject performed an IWR test to the limit of tolerance on each testing day. The same testing equipment was used. Each IWR test began with four minutes of cycling at $20 \mathrm{~W}$ (baseline), followed by a ramp increase in work rate at $20 \mathrm{~W}$ per minute (incremental response) to volitional exhaustion. This protocol was chosen such that the subjects would achieve a $\dot{\mathrm{V}}_{2} \mathrm{MAX}$ within 812 minutes, the optimal time period to achieve this. ${ }^{14}$ The subjects were asked to give maximal effort, but were informed that they could stop the test at any time.

After each CWR test, the subject was allowed to recover for at least 15 minutes, or until heart rate and $\dot{\mathrm{V}}_{2}$ returned to stable resting values, before proceeding to the next exercise test. To ensure a simple protocol for both subject and investigator, we chose to perform the CWR tests before the IWR test. During the latter, a subject is asked to exercise to exhaustion. We felt that this would adversely affect the subject's ability to produce a consistent exercise effort during the CWR test if performed after an exhausting IWR test. We chose a work rate that we felt would be below the subjects' gas exchange threshold based on gas exchange indices described elsewhere in the text.

\section{Data collection}

A metabolic measurement cart (2900; Sensormedics Corp Inc, Yorba Linda, California, USA) recorded breath by breath changes in minute ventilation, oxygen uptake, and carbon dioxide output. This system contains a mass flow meter for measurement of volume and discrete gas analysers for measurement of oxygen and carbon dioxide concentrations of expired air. Each breath was sampled at $100 \mathrm{~Hz}$ for volume, oxygen, and carbon dioxide concentrations, then displayed on a terminal and stored for analysis. To ensure quality control, the sensors were calibrated for volume and concentration before each test by repeated strokes of a precision built 3 litre syringe using two gas mixtures of known concentrations ( (a) $16 \% \mathrm{O}_{2}, 4 \% \mathrm{CO}_{2}$, and balance $\mathrm{N}_{2}$; (b) $26 \% \mathrm{O}_{2}, 0 \% \mathrm{CO}_{2}$, and balance $\mathrm{N}_{2}$ ). Lastly, each subject's heart rate and rhythm were monitored throughout the testing period using a three lead electrocardiograph (Hewlett-Packard; 78304A).

\section{Data analysis}

The classic four variables of aerobic fitness were determined from the breath by breath data: $\tau \dot{\mathrm{V}}_{2}, \dot{\mathrm{V}}_{2} \mathrm{MAX}, \dot{\mathrm{V}} \theta$, and $\eta$ as well as the slope of the $\tau \dot{\mathrm{V}}_{2} /$ work rate relation $\left(\Delta \dot{\mathrm{V}}_{2} / \Delta \dot{\mathrm{W}}\right)$. The method for determining $\tau \dot{\mathrm{V}}_{2}$ from the CWR tests used a non-linear regression analysis with a monoexponential model (BMDP, Cork, Ireland). Using this model, we determined what Karlsson et $a l^{16}$ termed the "mean response time" and what Gerbino et al ${ }^{16}$ termed the "effective time constant". We consider the term "effective time constant" most applicable to the results of our investigations. The effective time constant for oxygen uptake was calculated from each individual CWR test for both the on-transit and off-transit of exercise. We defined the terms on- $\tau \dot{\mathrm{V}} \mathrm{O}_{2}$ and off$\tau \dot{\mathrm{V}} \mathrm{O}_{2}$ to describe the effective time constants for the ontransit and off-transit of exercise respectively. In addition, the six individual CWR tests were time aligned and superimposed for both on-transit time and off-transit time and again used to calculate $\tau \dot{\mathrm{V}}_{2}$. These "pooled means" served as the yardstick.

For each CWR test, we established reference plateau values during the last minute of the $20 \mathrm{~W}$ baseline, the last two minutes of the $120 \mathrm{~W}$ steady state phase, and the last minute of the $20 \mathrm{~W}$ recovery. The steady state phase was scrutinised for evidence of upward drift, indicating that $\dot{\mathrm{V}}_{2}$ exceeded the gas exchange threshold. ${ }^{17}{ }^{18}$ We defined an unacceptable drift of $\Delta \tau \dot{\mathrm{V}}_{2}$ as exceeding $100 \mathrm{ml} / \mathrm{min}$ between three and six minutes of CWR exercise. ${ }^{17-19}$ In this manner, values for the time constant for both the on-transit and off-transit of 
exercise were derived by fitting the curve of the adaptive phase to the exponential function (fig 1, equation 1). The term "grand mean" will refer to the value obtained from averaging the individual CWR test results. As mentioned above, the time aligned and superimposed CWR tests derived from the pooled data served to derive the "pooled means".

The IWR tests served to calculate $\tau \dot{\mathrm{V}}_{2}$, as well as the $\dot{\mathrm{V}}_{2} \mathrm{MAX}, \dot{\mathrm{V}}_{2} \theta, \eta$, and the slope of the $\dot{\mathrm{V}}_{2}$ /work rate relation $\left(\Delta \dot{\mathrm{V}}_{2} / \Delta \dot{\mathrm{W}}\right)$. After the onset of exercise, the measured $\dot{\mathrm{V}}_{\mathrm{O}_{2}}$ does not match the ideal $\dot{\mathrm{V}}_{2}$, which would be expected for that instantaneous work rate if there were no oxygen deficit (fig 2). The slowed increase in $\dot{\mathrm{V}}_{2}$ is determined by the time constant. We used linear regression to define the slope of the $\dot{\mathrm{V}}_{2}$ response to incremental exercise taking care to be above the non-linear phase, which is influenced by the time constant, and also below the gas exchange threshold $\left(\dot{\mathrm{V}}_{2} \theta\right)$ so as to avoid any uncertainty relating to the influence of anaerobic metabolism on the slope. The range of data used for the regression analysis was from three minutes after the start of the ramp phase (which is considered sufficient time to achieve the linear response) to the metabolic threshold, which generally occurred between minutes five and six of exercise. The point of intersection of this slope with an extrapolation of the baseline $\dot{\mathrm{V}}_{2}$ defines $\tau \dot{\mathrm{V}}_{2}$ for the IWR test (fig 2), as described by Whipp et al. ${ }^{1}$ For the time constant derived from an IWR test, we used the term ramp $\tau \dot{\mathrm{V}}_{2}$.

The $\dot{\mathrm{V}} \mathrm{O}_{2} \mathrm{MAX}$ was selected as the highest $\dot{\mathrm{V}}_{2}$ seen within the last 30 seconds of exercise from a table of breath by breath data with nine-breath rolling averages. Two methods were used to determine $\dot{\mathrm{V}}_{2} \theta$ : (a) identification of an upward inflection in the plot of $\dot{\mathrm{V}}_{\mathrm{CO}_{2}}$ versus $\dot{\mathrm{V}}_{2}{ }^{20}{ }^{21} ;(b)$ identification of simultaneous upward inflections in the plots of $\dot{\mathrm{V}} \mathrm{E} / \dot{\mathrm{V}}_{2}$ and $\mathrm{PeTO}_{2}$ versus time while $\dot{\mathrm{V}} / \dot{\mathrm{V}}_{\mathrm{CO}}{ }_{2}$ and $\mathrm{PETCO}_{2}$ remained constant, the so called "dual criteria". ${ }^{22}$ The relevant plots were analysed in a blind review by two exercise physiologists with 25 years of combined experience in this field.

\section{Statistical analysis}

We used standard statistical methods ${ }^{23} 24$ including descriptive statistics (group means and standard deviations), linear regression, and limits of agreement. $p \leqslant 0.05$ was considered significant.

\section{Quality assurance}

Before the start of the study, we validated the metabolic system against standard Douglas bag techniques. ${ }^{25}$ We used a

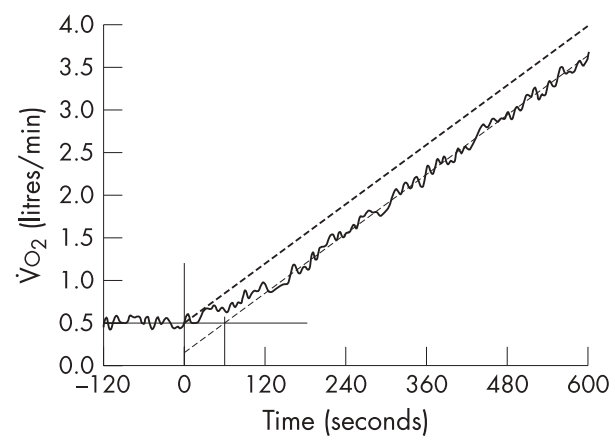

Figure 2 Progressive increase in $\mathrm{V}_{2}$ in response to incremental work rate exercise: a geometric analysis is superimposed on the data. After an initial baseline is established, a progressive increase in $\mathrm{O}_{2}$ uptake should occur with the onset of incremental exercise (ideal $\mathrm{V}_{2}$ ). However, at any moment in time, the measured $\mathrm{V}_{2}$ does not match the ideal $\dot{\mathrm{V}}_{2}$ for that instantaneous work rate. Rather, the rate of increase is slowed by the time constant. Linear regression defines the slope of the response. Geometric analysis proves that the point of intersection of this slope with extrapolation to the baseline defines $\tau$. mass spectrometer to determine relevant gas concentrations within the collection bags. We calibrated the cycle ergometer with a dynamic torque meter, which measures braking resistance at the cycle crank such that braking resistance was determined at the cycle crank arms.

\section{RESULTS}

\section{Subjects}

Five of the subjects yielded 30 CWR tests and 15 IWR tests for analysis. The mean (SD) age, height, and weight of the subjects were 27 (5) years, $1.77(0.10) \mathrm{m}$, and 77 (9) $\mathrm{kg}$ respectively. Table 1 lists exercise capacity expressed as $\dot{\mathrm{V}} \mathrm{O}_{2} \mathrm{MAX}$, oxygen uptake as percentage of predicted maximum (\%pred $\left.\dot{\mathrm{V}}_{2} \mathrm{MAX}\right),{ }^{26}$ the metabolic or gas exchange threshold $\left(\dot{\mathrm{V}}_{2} \theta\right)$, and oxygen uptake at steady state $\left(\dot{\mathrm{V}}_{2} \mathrm{SS}\right)$ for each subject. Their mean (SD) values were: $3.84(0.44)$ litres/min, $115(13) \%, 1.88$ (0.23) litres/min, and $1.67(0.07)$ litres/min, respectively. The remaining five subjects were dropped from the analysis because of a continuing upward drift in oxygen uptake throughout the CWR tests, as defined in the Methods section. The characteristics of the excluded subjects were: age 23 (3) years, height $1.79(0.08) \mathrm{m}$, weight $78.0(8.0) \mathrm{kg}$, $\dot{\mathrm{V}}_{2}$ MAX 3.12 (0.15) litres/min,\%pred $\dot{\mathrm{V}}_{2} \mathrm{MAX} 89$ (3)\%, $\dot{\mathrm{V}}_{2} \theta$ 1.59 (0.21) litres/min, and $\dot{\mathrm{V}}_{2} \mathrm{ss} 1.83$ (0.05). Thus, the excluded subjects were slightly younger and anthropometrically matched but less well conditioned as manifested by lower $\dot{\mathrm{V}}_{2} \mathrm{MAX}$ and $\dot{\mathrm{V}}_{2} \theta$.

\section{Reproducibility of $\tau \dot{\mathrm{V}} \mathrm{O}_{2}$ from CWR tests}

Tables 2 and 3 list the values determined from the analysis of individual CWR tests for $\tau \dot{\mathrm{V}}_{2}$ for both on-transit and offtransit of exercise using non-linear regression with a monoexponential model. When we came to analyse the test results, we found that one off-transit value was corrupted by the electronic data storage system (table 3 ). We continued the analysis with this data point missing. Table 4 summarises the analysis of mean values. The data obtained show that the grand and pooled means were nearly identical for both on$\tau \dot{\mathrm{V}}_{2}$ (38.1 and 38.0 seconds respectively) and off- $\tau \dot{\mathrm{V}}_{\mathrm{O}_{2}}(39.2$ and 39.0 seconds respectively). Although we acknowledge some variability in the results for individual subjects-for example, on- $\tau \dot{\mathrm{V}}_{2}$ for subject 1 ranged from 34.0 to 44.3 seconds - the within subject coefficients of variability for both on- $\tau \dot{\mathrm{V}}_{2}$ (11.5\%) and off- $\tau \dot{\mathrm{V}}_{\mathrm{O}_{2}}$ (12.6\%) fell within the range of acceptability for physiological testing; a coefficient of variability of less than $15 \%$ is generally considered acceptable for clinical testing. For instance, the American Thoracic Society criteria for pulmonary function testing allow 12-15\% variability in most variables. ${ }^{27}$ The between subject coefficient of variability was $14.7 \%$ for on- $\tau \dot{\mathrm{V}} \mathrm{O}_{2}$ and $12.4 \%$ for off- $\tau \dot{\mathrm{V}} \mathrm{O}_{2}$. Furthermore, the standard deviation for $\tau \dot{\mathrm{V}}_{2}$ was only two to six seconds, which probably does not represent any clinically meaningful alteration in aerobic performance.

\section{Comparison of on $-\tau \dot{\mathrm{V}} \mathrm{O}_{2}$ with off- $\tau \dot{\mathrm{V}} \mathrm{O}_{2}$}

Figure 3 depicts this relation showing a significant correlation $(r=0.870)$. The data indicate that on- $\tau \dot{V}_{O_{2}}$ and off- $\tau \dot{V}_{O_{2}}$ are approximately equal when calculated from CWR tests. To test the validity of deriving $\tau \dot{\mathrm{V}}_{2}$ from combined on- and offtransits for a series of tests, we compared the grand mean and $95 \%$ confidence intervals for $\tau \dot{\mathrm{V}}_{2}$ derived by averaging on- $\tau \dot{\mathrm{V}}_{2}$ and off- $\tau \dot{\mathrm{V}}_{2}$ for the first three tests in each subject with the grand mean and $95 \%$ confidence intervals for $\tau \dot{\mathrm{V}}_{2}$ derived from six on-transits and also six off-transits in the same subjects. For three sets of on- and off-transits, $\tau \dot{\mathrm{V}}_{2}$ was 39.2 seconds (95\% confidence interval (CI) 30.9 to 47.5 ) whereas for six on-transits $\tau \dot{\mathrm{V}} \mathrm{O}_{2}$ was 38.1 seconds (95\% CI 28.0 to 48.2 ) and for six off-transits $\tau \dot{\mathrm{V}}_{2}$ was 39.0 seconds (95\% CI 30.6 to 47.4). Thus the confidence interval for $\tau \dot{\mathrm{V}}_{2}$ 
Table 1 Subject characteristics and exercise capacity

\begin{tabular}{|c|c|c|c|c|c|c|c|c|c|c|c|}
\hline Subject & $\begin{array}{l}\text { Age } \\
\text { (years) }\end{array}$ & $\begin{array}{l}\text { Height } \\
\text { (m) }\end{array}$ & $\begin{array}{l}\text { Weight } \\
\text { (kg) }\end{array}$ & $\begin{array}{l}\dot{\mathrm{V}} \mathrm{O}_{2} \mathrm{MAX} \\
\text { (litres/min) }\end{array}$ & $\begin{array}{l}\text { \%pred } \\
\dot{\text { Vo }}{ }_{2} \operatorname{MAX}\end{array}$ & $\begin{array}{l}\dot{\mathrm{V}} \mathrm{O}_{2} \theta \\
\text { (litres/min) }\end{array}$ & $\begin{array}{l}\dot{\mathrm{V}} \mathrm{O}_{2} \mathrm{ss} \\
\text { (litres/min) }\end{array}$ & $\begin{array}{l}\dot{\mathrm{V}} \mathrm{o}_{2} \mathrm{ss} / \\
\dot{\mathrm{Vo}}{ }_{2} \theta(\%)\end{array}$ & $\begin{array}{l}\dot{\mathrm{V}} \mathrm{o}_{2} \theta / \\
\dot{\mathrm{V}} \mathrm{o}_{2} \operatorname{MAX}(\%)\end{array}$ & $\Delta \dot{\mathrm{V}}_{2} / \Delta \dot{\mathrm{W}}$ & $\eta(\%)$ \\
\hline 1 & 25 & 1.70 & 68 & 3.44 & 101 & 1.75 & 1.63 & 0.93 & 51 & 9.9 & 29.1 \\
\hline 2 & 21 & 1.78 & 77 & 4.06 & 115 & 1.87 & 1.72 & 0.92 & 53 & 10.3 & 28.0 \\
\hline 3 & 31 & 1.93 & 80 & 3.29 & 102 & 1.69 & 1.59 & 0.94 & 53 & 10.2 & 28.3 \\
\hline 4 & 33 & 1.68 & 70 & 4.09 & 130 & 2.08 & 1.66 & 0.80 & 66 & 10.9 & 26.4 \\
\hline 5 & 24 & 1.78 & 91 & 4.33 & 126 & 2.03 & 1.77 & 0.87 & 59 & 11.3 & 25.5 \\
\hline Mean & 27 & 1.77 & 77 & 3.84 & 115 & 1.88 & 1.67 & 0.89 & 56 & 10.5 & 27.0 \\
\hline SD & 5 & 0.10 & 9 & 0.44 & 13 & 0.23 & 0.07 & 0.06 & 6 & 0.9 & 1.6 \\
\hline
\end{tabular}

The values derived from incremental exercise testing are the means of three tests for each subject. $\Delta \dot{\mathrm{V}} \mathrm{O}_{2} / \Delta \dot{\mathrm{W}}$ is the slope of the relation between oxygen uptake and work rate. $\eta$ is the work efficiency derived from $\Delta \dot{V}_{O_{2}} / \Delta \dot{W}$ using the formula: $\eta=288\left(\Delta \dot{V}_{2} / \Delta \dot{W}\right) .{ }^{6}$ See text for explanation of other symbols and abbreviations.

was actually smaller when combining values from both the on- and off-transits.

\section{Similarity of the grand mean and pooled mean values for $\tau \dot{\mathrm{V}} \mathrm{O}_{2}$}

For each subject, we compared the grand mean $\tau \dot{\mathrm{V}}_{2}$, derived by averaging the individual values of $\tau \dot{\mathrm{V}}_{2}$ derived from the six CWR tests, with the pooled mean $\tau \dot{\mathrm{V}}_{2}$, derived by time alignment and superimposition of the data from all of the six CWR tests. The values for each subject were in excellent agreement (tables 2 and 3). Figure 4 shows a plot of grand mean $\tau \dot{\mathrm{V}}_{\mathrm{O}_{2}}$ versus pooled mean $\tau \dot{\mathrm{V}}_{2}$ for all subjects. The correlation coefficient for this relation was 0.997. Although it is clear that the two methods of analysis arrive at similar values for $\tau \dot{\mathrm{V}}_{2}$, the confidence interval for the pooled mean $\tau \dot{\mathrm{V}} \mathrm{O}_{2}$ was consistently tighter, which would slightly favour time alignment and superimposition of data as the method of choice for deriving the time constant.

\section{Accuracy of individual CWR tests}

To provide a method to assess the accuracy of individual CWR tests, the $95 \%$ confidence intervals for the pooled means for both on- $\tau \dot{\mathrm{V}}_{2}$ and off- $\tau \dot{\mathrm{V}}_{2}$ were determined for each subject. As shown in table 5, the time constant fell within the 95\% confidence interval of the pooled mean in only three of the five subjects during the first CWR test for both on- $\tau \dot{\mathrm{V}}_{2}$ and off- $\tau \dot{\mathrm{V}} \mathrm{O}_{2}$. This yielded a combined accuracy of $60 \%$ after one test. However, after the first four tests, the average values for all five subjects fell within their respective 95\% confidence intervals for both on- $\tau \dot{\mathrm{V}} \mathrm{O}_{2}$ and off- $\tau \dot{\mathrm{V}}_{2}$, yielding $100 \%$ accuracy at this point. Alternatively, when we combined and averaged the on- $\tau \dot{\mathrm{V}}_{2}$ and off- $\tau \dot{\mathrm{V}} \mathrm{O}_{2}$ values for each test, we found all subjects to be within the 95\% confidence interval by the second test (table 5 ). We define sequential averaging as the rolling average of each test in sequence from the first to the final value.

\section{Comparison between IWR and CWR tests}

Geometric analysis was used to determine $\tau \dot{\mathrm{V}}_{2}$ from the IWR tests. Table 6 lists the values for each subject. As seen in table 4 , all of the mean (SD) ramp- $\tau \dot{\mathrm{V}}_{2}$ values were substantially higher than both the on- $\tau \dot{\mathrm{V}} \mathrm{O}_{2}$ and off- $\tau \dot{\mathrm{V}} \mathrm{O}_{2}$ for CWR tests: 60.8 (15.4), 38.1 (5.6), and 39.2 (4.9) seconds respectively. Figure 5 illustrates a comparison of the average ramp- $\tau \dot{V}_{O_{2}}$ versus the on- $\tau \dot{V}_{O_{2}}$ for each subject showing that the ramp value was consistently longer. Table 4 also shows the coefficient of variability for the ramp- $\tau \dot{V}_{O_{2}}$ values. The within subject and between subject values were both equally high at $21.0 \%$ and $25.4 \%$ respectively, unlike the lower within subject coefficient of variability for CWR tests.

\section{Other variables of aerobic fitness}

Table 1 lists the mean values and standard deviations for $\Delta \dot{\mathrm{V}}_{2} / \Delta \dot{\mathrm{W}}$ for each subject as derived from the three IWR tests. The overall mean (SD) for all subjects was $10.5(0.9)$ $\mathrm{ml} / \mathrm{min} /$ watt. The work efficiency was found to be 27.0 (1.6)\%. We compared on- $\tau \dot{\mathrm{V}}_{2}$ and $\dot{\mathrm{V}}_{2} \mathrm{MAX}$, finding a close correlation $(r=0.73)$, which illustrated that more physically fit subjects (with a higher $\dot{\mathrm{V}}_{2} \mathrm{MAX}$ ) had faster time constants. We also compared on- $\tau \dot{\mathrm{V}}_{2}$ and $\dot{\mathrm{V}}_{2} \theta$, again finding a close correlation $(r=0.89)$, which further supports the understanding that $\tau \dot{\mathrm{V}}_{2}$ is a useful measure of physical fitness.

\section{DISCUSSION}

Aerobic performance is a means of evaluating fitness or the extent of cardiopulmonary disease. Three important variables of aerobic function $\left(\dot{\mathrm{V}}_{2} \mathrm{MAX}, \dot{\mathrm{V}}_{2} \theta\right.$, and $\left.\tau \dot{\mathrm{V}}_{2}\right)$ may be assessed and followed over time to evaluate the responses to exercise training or the progression of disease. The fourth, $\eta$, reflects basic biochemical energy-yielding reactions needed for muscle contraction and is similar for young or old, male or female, and trained or untrained people. ${ }^{28}{ }^{29}$ An IWR protocol would seem to be more valuable than the CWR test by providing all four variables including $\dot{\mathrm{V}}_{2} \mathrm{MAX}$, which is considered the best metabolic index of the work capacity of a given individual. ${ }^{30}$ However, in patients with major cardiopulmonary disease and sometimes in healthy subjects, the peak $\dot{\mathrm{VO}}_{2}$ measured at the end of an incremental test may be influenced by motivation, subjective evaluation of the clinician in assessing the end point of exercise, or safety issues. By contrast, the CWR test is submaximal, potentially safer, and influenced less by the motivation of the subject. The important variable

Table 2 On- $\tau \dot{\mathrm{V}}_{2}$ for constant work rate tests in seconds

\begin{tabular}{|c|c|c|c|c|c|c|c|c|c|c|c|}
\hline Subject & 1 & 2 & 3 & 4 & 5 & 6 & Grand mean & SD & CV (\%) & Pooled mean & LOA (\%) \\
\hline 1 & 42.9 & 37.4 & 39.2 & 35.8 & 44.3 & 34.0 & 38.9 & 4.0 & 10.4 & 38.9 & 20.2 \\
\hline 2 & 38.5 & 34.6 & 43.7 & 36.4 & 41.8 & 37.5 & 38.8 & 3.4 & 8.8 & 38.6 & 17.2 \\
\hline 3 & 44.6 & 44.8 & 47.3 & 42.5 & 45.4 & 47.6 & 45.4 & 1.9 & 4.1 & 45.5 & 8.2 \\
\hline 4 & 38.9 & 33.3 & 30.9 & 29.0 & 27.8 & 26.3 & 31.0 & 4.6 & 14.7 & 30.7 & 29.1 \\
\hline 5 & 25.5 & 45.6 & 40.0 & 36.9 & 34.6 & 36.4 & 36.5 & 6.6 & 18.1 & 36.4 & 35.4 \\
\hline Mean & 38.1 & 39.1 & 40.2 & 36.1 & 38.8 & 36.3 & 38.1 & & & 38.0 & \\
\hline SD & 7.5 & 5.7 & 6.1 & 4.8 & 7.5 & 7.7 & 5.6 & & & 5.3 & \\
\hline
\end{tabular}

CV, Coefficient of variability; LOA, limits of agreement 
Table 3 Off- $\tau \dot{V}_{2}$ for constant work rate tests in seconds

\begin{tabular}{|c|c|c|c|c|c|c|c|c|c|c|c|}
\hline Subject & 1 & 2 & 3 & 4 & 5 & 6 & Grand mean & SD & CV (\%) & Pooled mean & LOA (\%) \\
\hline 1 & 32.2 & 38.3 & 59.6 & 41.9 & 42.1 & 42.1 & 42.7 & 9.1 & 21.3 & 41.9 & 41.7 \\
\hline 2 & 41.7 & 37.8 & 40.5 & 38.4 & 39.3 & 39.3 & 39.5 & 1.4 & 3.7 & 39.5 & 6.9 \\
\hline 3 & 40.9 & 47.1 & 42.4 & 40.4 & 42.8 & 40.0 & 42.3 & 2.6 & 6.2 & 42.3 & 12.1 \\
\hline 4 & 31.8 & 30.9 & 34.8 & 28.9 & 31.1 & * & 31.5 & 2.1 & 6.8 & 31.6 & 13.1 \\
\hline 5 & 36.8 & 35.7 & 38.7 & 37.6 & 43.7 & 48.8 & 40.2 & 5.0 & 12.5 & 39.9 & 24.4 \\
\hline Mean & 36.7 & 37.9 & 43.2 & 37.4 & 39.8 & 42.6 & 39.2 & & & 39.0 & \\
\hline SD & 4.7 & 5.9 & 9.6 & 5.1 & 5.1 & 4.4 & 4.9 & & & 4.3 & \\
\hline
\end{tabular}

obtained from a CWR test is the time constant for oxygen uptake. In patients with moderate cardiopulmonary dysfunction, serial constant work rate tests may be easily tolerated, and changes in $\tau \dot{\mathrm{V}}_{2}$ may be measured over time. ${ }^{80}{ }^{20} 11$ The determination of $\tau \dot{\mathrm{V}}_{2}$ for an IWR test and a single CWR test has not been validated against the "gold standard" of multiple repetitions of CWR tests. For this reason, we sought to develop and validate a simple non-invasive technique with the potential for clinical utility.

We found $\tau \dot{\mathrm{V}}_{2}$ to be a reproducible variable of aerobic fitness from the CWR protocol. The mean (SD) value for our healthy untrained male subjects was 38.1 (5.6) seconds for on-transit and 39.2 (4.9) seconds for off-transit of exercise, which was within the reported range of normal values for this type of subject. ${ }^{13-51132}$ Yoshida and Whipp ${ }^{4}$ reported quite similar values for on- $\tau \dot{\mathrm{V}}_{2}$ and off- $\tau \dot{\mathrm{V}}_{2}$ in six subjects, but felt it was necessary to repeat the CWR protocol at least eight times in each of their subjects to optimise the signal/ noise ratio. In our study, in which each subject performed six repetitions of the CWR protocol, the within subject coefficient of variability was $11.5 \%$ and $12.6 \%$ for the on-transit and offtransit respectively. Arguably, these values lie within an acceptable range for human physiological measurements. The mean values for on- $\tau \dot{\mathrm{V}}_{2}$ and off- $\tau \dot{\mathrm{V}} \mathrm{O}_{2}$ were within four seconds of each other for each of the experimental subjects. Furthermore, on- $\tau \dot{\mathrm{V}}_{2}$ and off- $\tau \dot{\mathrm{V}}_{2}$ correlated well with each other $(r=0.870$; fig 3$)$. Admittedly this correlation is strongly influenced in this study by the one subject with fast $\dot{\mathrm{V}}_{2}$ kinetics. Examining the other four subjects, one sees that off- $\tau \dot{\mathrm{V}} \mathrm{O}_{2}$ did not differ considerably whereas on- $\tau \dot{\mathrm{V}}_{2}$ varied by as much as 10 seconds between subjects. A correlation between on- $\tau \dot{\mathrm{V}}_{2}$ and off- $\tau \dot{\mathrm{V}}_{2}$ has been previously shown, ${ }^{3432}{ }^{33}$ consistent with the hypothesis that these two measures are representative of a similar physiological entity. Other investigators have shown similarity between on and off kinetics for moderate intensity exercise (comparable to the work rates used in this study), but have shown dynamic asymmetries between on and off kinetics for heavy and very heavy exercise (essentially above the metabolic threshold for blood lactate accumulation). ${ }^{34}$

Our analysis shows, interestingly, that a valid measure of $\tau \dot{\mathrm{V}}_{2}$ can be derived either by time alignment and superimposition of data from repeated CWR tests, the pooled mean, or by averaging on- $\tau \dot{\mathrm{V}}_{2}$ and off- $\tau \dot{\mathrm{V}}_{\mathrm{O}_{2}}$ from sequential tests, the grand mean. Although the confidence intervals for the pooled means were consistently, but only slightly, narrower than for the grand means, the similarity of the results validates both approaches for the derivation of $\tau \dot{\mathrm{V}}_{2}$. In three out of five of our subjects, the value for $\tau \dot{\mathrm{V}}_{2}$ from the first individual test was within the $95 \%$ confidence interval of the pooled value for both on-transit and offtransit. By the fourth test, the average value for $\tau \dot{\mathrm{V}}_{2}$ fell within the $95 \%$ confidence interval of the pooled value in all cases. This implies that only four tests are needed to obtain acceptable accuracy in our type of subject. Alternatively, by sequentially averaging the values for on- $\tau \dot{\mathrm{V}} \mathrm{O}_{2}$ and off- $-\tau \dot{\mathrm{V}} \mathrm{O}_{2}$ together, we found all five subjects to be within the $95 \%$ confidence interval of the pooled value by the second CWR test.

We further assessed the validity of combining both on- $\tau \dot{\mathrm{V}}_{2}$ and off- $\tau \dot{\mathrm{V}} \mathrm{O}_{2}$, by doing so for the first three tests in each subject and comparing the mean value for $\tau \dot{\mathrm{V}}_{2}$ with that obtained by combining six on-transits or alternatively six offtransits (pooled means). These three approaches gave similar values for $\tau \dot{\mathrm{V}}_{2}$, with the confidence interval derived by combining both on-transits and off-transits actually being smaller than for the other two methods. The means (95\% CI) were 39.2 (30.9 to 47.5) seconds for combined on- $\tau \dot{\mathrm{V}}_{2}$ and off- $\tau \dot{\mathrm{V}}_{2}$ from three tests, 38.0 (27.6 to 48.4) seconds for six on-transits, and 39.0 (30.6 to 47.4) seconds for six offtransits. We can conclude from these findings that the method of monoexponential non-linear regression analysis of three individual on-transits and off-transits with simple averaging of all of the results is just as valid as the traditional approach of time alignment and superimposition of the data from either the on-transits or the off-transits of six studies.

The reduction in the necessity for repetitions emphasises the utility of our methodology. Lamarra et a ${ }^{35}$ studied oxygen uptake kinetics for CWR transitions from 0 to $100 \mathrm{~W}$ in five normal subjects, where $0 \mathrm{~W}$ represented unloaded pedalling. They derived an equation for the number of repetitions required for a desired confidence interval for $\tau \dot{\mathrm{V}} \mathrm{O}_{2}$. They suggested that only analysing the on-transit for this work rate protocol required between three and eight transitions to achieve a 95\% confidence interval of two seconds for $\tau \dot{\mathrm{V}}_{2}$ The need to repeat a clinical test is disadvantageous in its application to clinical practice. This shortcoming is true of other testing variables. For example, the timed walking test

Table 4 Comparison of mean values for $\tau \dot{\mathrm{V}} \mathrm{O}_{2}$

\begin{tabular}{|c|c|c|c|c|c|c|c|}
\hline & \multicolumn{2}{|c|}{ Mean (seconds) } & \multicolumn{2}{|c|}{ Within subjects } & \multicolumn{3}{|c|}{ Between subjects } \\
\hline & Pooled & Grand & $\bar{s}$ & $\mathrm{CV}(\%)$ & 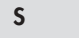 & CV (\%) & LOA (\%) \\
\hline $\mathrm{On}-\tau \dot{\mathrm{V}}_{2}$ & $38.0(5.3)$ & $38.1(5.6)$ & 19.2 & 11.5 & 31.3 & 14.7 & 28.8 \\
\hline Off- $-\tau \mathrm{V}_{2}$ & $39.0(4.3)$ & $39.2(4.9)$ & 24.4 & 12.6 & 23.6 & 12.4 & 24.5 \\
\hline Ramp $\tau \hat{V}_{2}$ & - & 60.8 (15.4) & 163.0 & 21.0 & 238.3 & 25.4 & 49.6 \\
\hline
\end{tabular}




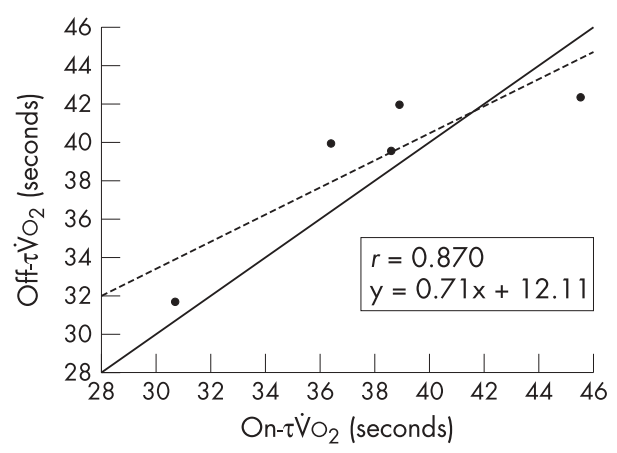

Figure 3 Correlation of on- $\tau \dot{\mathrm{V}} \mathrm{O}_{2}$ and off- $\tau \dot{\mathrm{V}}_{2}$. These two values show a close correlation implying the measurement of similar physiological phenomena.

used in cardiopulmonary disease evaluation and rehabilitation is known to have a learning effect for at least the first three tests. ${ }^{36}$ Our methodology for determining $\tau \dot{\mathrm{V}} \mathrm{O}_{2}$ may circumvent unnecessary repetitions and facilitate its application to clinical testing. Admittedly we have tested normal subjects who allowed selection of a relatively large $\Delta \tau \dot{\mathrm{V}} \mathrm{O}_{2}$. The study of clinical populations remains more challenging, but the simultaneous measurement and averaging of on- $\tau \dot{\mathrm{V}}_{2}$ and off- $\tau \dot{\mathrm{V}}_{2}$ could still be of value.

We have shown the reliability as well as the utility of $\tau \dot{\mathrm{V}}_{2}$ as a variable of aerobic fitness by comparing $\tau \dot{\mathrm{V}}_{2}$ with $\dot{\mathrm{V}}_{2}$ MAX and with $\dot{\mathrm{V}}_{2} \theta$. We showed that the subjects with shorter time constants for oxygen uptake were able to achieve higher values of maximum oxygen uptake and exhibited higher gas exchange thresholds. On- $\tau \dot{\mathrm{V}}_{2}$ and $\dot{\mathrm{V}}_{2} \mathrm{MAX}$ were directly correlated $(r=0.73)$, whereas on- $\tau \dot{\mathrm{V}} \mathrm{O}_{2}$ and $\dot{\mathrm{V}}_{2} \theta$ were inversely correlated $(r=0.89)$. The stronger correlation with $\dot{\mathrm{V}}_{2} \theta$ may indicate similar reliability and validity of these two submaximal measures and underscores their potential for clinical application. Chilibeck et al ${ }^{32}$ reported that $\tau \dot{\mathrm{V}}_{2}$ correlated significantly with $\dot{\mathrm{V}}_{2} \mathrm{MAX}$. Other investigators have reported that the half time for $\dot{\mathrm{V}}_{2}$ uptake correlates well with $\dot{\mathrm{V}}_{2} \mathrm{MAX}$ in highly trained subjects. ${ }^{37}$ The study of Chilibeck $e$ al $^{32}$ reported that older subjects with a $\dot{\mathrm{V}}_{2} \mathrm{MAX}$ of about $25 \mathrm{ml} / \mathrm{kg} / \mathrm{min}$ had $\tau \dot{\mathrm{V}}_{2}$ ranging from 40 to 70 seconds, whereas younger subjects with a $\tau \dot{\mathrm{V}}_{2} \mathrm{MAX}$ of about $45 \mathrm{ml} / \mathrm{kg} /$ min had $\tau \dot{\mathrm{V}}_{2}$ ranging from 20 to 45 seconds. Although their subjects were of similar age to ours, their $\tau \dot{\mathrm{V}}_{2}$ values varied widely compared with our subjects. Our ability to show strong correlations between $\tau \dot{\mathrm{V}}_{2}$ and $\dot{\mathrm{V}}_{\mathrm{O}_{2} \mathrm{MAX}}$ and $\dot{\mathrm{V}}_{2} \theta$ in a relatively homogeneous group of subjects actually lends strength to our chosen methodology.

In contrast with the CWR protocol, we found $\tau \dot{\mathrm{V}} \mathrm{O}_{2}$ from the IWR test to be less reliable and less reproducible. We calculated the mean (SD) value for ramp- $\tau \dot{\mathrm{V}}_{2}$ to be 60.8 (15.4) seconds with a within subject coefficient of variability of $21.0 \%$ (table 4 ). An unacceptably high coefficient of variability for oxygen uptake kinetics has been previously reported for IWR tests. ${ }^{10}$ When comparing ramp- $\tau \dot{\mathrm{V}} \mathrm{O}_{2}$ with on- $\tau \dot{\mathrm{V}}_{2}$, the ramp values were systematically longer (fig 5 ). This finding contrasts with that of Whipp et al ${ }^{1}$ who described similar values for $\tau \dot{\mathrm{V}}_{2}$ derived from a single short duration (eight minutes) ramp test and single CWR test (at a work rate of $80 \%$ of $\dot{\mathrm{V}}_{2} \theta$ for five to six minutes from a baseline of unloaded pedalling). Interestingly, the two studies used similar types of subjects but produced different $\tau \dot{\mathrm{V}}_{2}$ values for the on-transit of exercise in CWR tests: 48.7 (8.9) seconds for Whipp et al and 38.1 (5.6) seconds for our subjects (mean (SD) values). Theoretically, the physiological determinants of the time constant from a CWR test and an IWR test should be identical. However, mechanistically they might be different

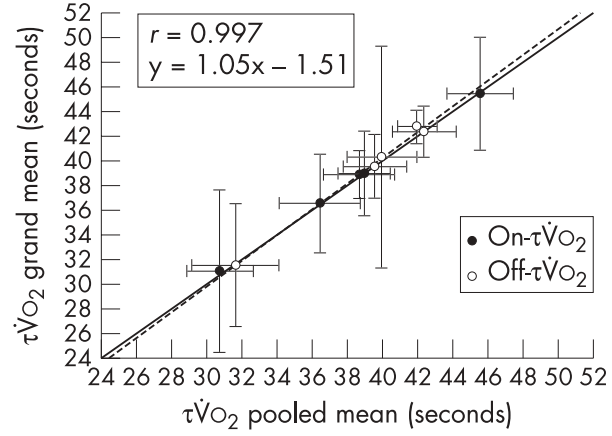

Figure 4 Correlation between the grand mean values for on- $\tau \dot{\mathrm{V}}_{2}$ and off- $\tau \dot{\mathrm{V}} \mathrm{O}_{2}$, derived by arithmetic averaging of $\tau \dot{\mathrm{V}} \mathrm{O}_{2}$ from six individual exercise transitions, and the pooled mean values for on $-\tau \mathrm{V}_{2}$ and off$\tau \grave{\mathrm{V}}_{2}$, derived by time alignment and superimposition of the data from six individual exercise transitions. The correlation coefficient and closeness to the line of identity (not shown) reflects that the two methods derive virtually identical results.

because, by definition, an IWR test never achieves a steady state. Perhaps the ever increasing work rate influences the continuing dynamic phase of cardiovascular adjustment, thus affecting the measurement of $\tau \dot{\mathrm{V}}_{2}$ by this approach. Furthermore, inclusion of data above the metabolic or gas exchange threshold could adversely influence the geometric analysis used to derive ramp- $\tau \dot{\mathrm{V}}_{2}$. We were careful to avoid this potential problem by defining the slope of the response above the time delay phase and below the gas exchange threshold. Further investigation is warranted to elucidate these mechanistic differences.

Another objective of our study was to design a protocol that would optimise the signal/noise ratio and facilitate interpretation. Firstly, we attempted to select a work rate that was as high as possible $(120 \mathrm{~W})$ yet remained below the metabolic or gas exchange threshold. Secondly, we chose a relatively high baseline $(20 \mathrm{~W})$ to minimise the noise often observed at rest or at very low work rates. We have unpublished data from our laboratory in normal subjects that indicate that the coefficient of variability for $\dot{\mathrm{V}}_{2}$ at rest is about $37 \%$ ( $\mathrm{SD}=96 \mathrm{ml} / \mathrm{min}$ ) whereas for this study, the coefficient of variability for $\dot{\mathrm{V}}_{2}$ at a baseline of $20 \mathrm{~W}$ was $23 \%$ ( $\mathrm{SD}=158 \mathrm{ml} / \mathrm{min}$ ). Lamarra et al $^{35}$ studied breath by breath fluctuations in $\dot{\mathrm{V}}_{2}$ for CWR transitions from 0 to $100 \mathrm{~W}$. They reported that the standard deviation of the "noise" was independent of metabolic rate and about $10 \%$ of the change in $\dot{\mathrm{V}}_{2}$ between the two CWRsthat is, about $90 \mathrm{ml} / \mathrm{min}$. Thus the "noise" in our data was similar to that reported by Lamarra et al but shown to be proportionally smaller at the higher metabolic rate. Importantly, Lamarra et al showed that the accuracy of $\tau \dot{\mathrm{V}}_{2}$ derived by non-linear least squares estimation was directly proportional to the standard deviation of the noise. In other words, as we hypothesised, a method such as the one we adopted, which reduces noise, should lead to greater accuracy in the determination of $\tau \dot{\mathrm{V}}_{2}$.

Table 5 Accuracy of $\tau \dot{\mathrm{V}} \mathrm{O}_{2}$ shown by the number of test results falling within the $95 \%$ confidence interval of the pooled mean

\begin{tabular}{llll}
\hline $\begin{array}{l}\text { Sequential } \\
\text { average of tests }\end{array}$ & On- $\tau \dot{\mathrm{V}} \mathrm{o}_{2}$ & Off- $\tau \dot{\mathrm{V}} \mathrm{o}_{2}$ & $\begin{array}{l}\left(\mathrm{On}-\tau \dot{\mathrm{V}} \mathrm{o}_{2}+\right. \\
\left.\text { off- } \tau \mathrm{V}_{2}\right) / 2\end{array}$ \\
\hline 1 & $3(60 \%)$ & $3(60 \%)$ & $3(60 \%)$ \\
2 & $4(80 \%)$ & $4(80 \%)$ & $5(100 \%)$ \\
3 & $4(80 \%)$ & $5(100 \%)$ & \\
4 & $5(100 \%)$ & & \\
\hline
\end{tabular}




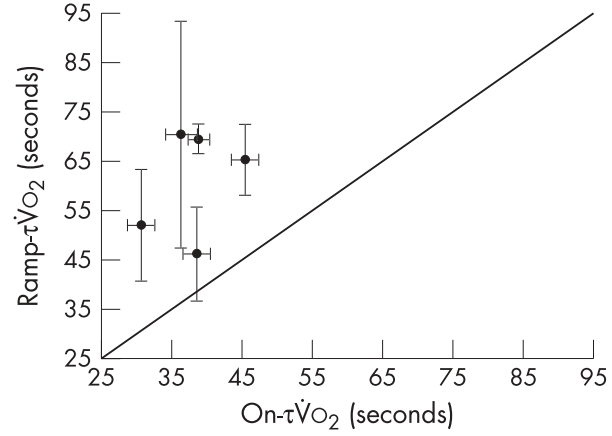

Figure 5 Correlation of on- $\tau \dot{\mathrm{V}} \mathrm{O}_{2}$ and ramp- $\tau \mathrm{V}_{2}$ The diagonal line is the line of identity. Ramp- $\tau \dot{\mathrm{V}} \mathrm{O}_{2}$ was systematically longer than on- $\tau \dot{\mathrm{V}} \mathrm{O}_{2}$.

Oxygen uptake kinetics during constant load exercise of moderate intensity is defined by three phases. ${ }^{38}$ The first is characterised by the immediate increase in gas exchange at the start of exercise, lasting for about 15-20 seconds, and has been attributed to an abrupt increase in pulmonary blood flow. The second phase exhibits a monoexponential rise in $\dot{\mathrm{V}}_{2}$, described by $\tau \dot{\mathrm{V}}_{2}$, reaching a new steady state by three minutes for exercise below the gas exchange threshold. ${ }^{58} \mathrm{We}$ chose a high baseline, rather than beginning at rest or with unloaded pedalling to eliminate or minimise any phase I effect. This allowed us to apply a monoexponential model to the overall response, thus simplifying the measurement of the time constant for phase II. ${ }^{12}$ Although they incorporated a transit delay factor, Lamarra $e a^{35}$ also advocated the use of a first order model to describe the kinetic response. We are not proposing that a $100 \mathrm{~W}$ step in work rate is appropriate for clinical measurement, but rather that a desirable approach would aim to select the highest below-threshold $\dot{\mathrm{V}}_{2}$ for a given individual, thus obtaining steady state conditions with the greatest possible $\Delta \dot{\mathrm{V}}_{2}$. A standard approach such as this would also allow some comparison among different subjects.

During exercise above the metabolic or gas exchange threshold, characteristic changes occur: the $\dot{\mathrm{V}}_{2}$ kinetics change by a second exponential function described by a slow component with a longer time constant ${ }^{59}$ associated with a progressive increase in blood lactate ${ }^{17}$ and a progressive upward drift of $\dot{\mathrm{V}}_{2} \cdot{ }^{39}$ Different protocols have been reported describing the work rate in normal subjects above which this drift occurs, ranging from $120 \mathrm{~W}^{40}$ to more than $150 \mathrm{~W} .{ }^{5}$ We chose a CWR at the lower end of this range, yet still found that a significant number of subjects had evidence of $\dot{\mathrm{V}}_{2}$ drift based on our stringent criterion of $>100 \mathrm{ml} / \mathrm{min}$ between three and six minutes of the CWR exercise. The CWR above which the drift may be expected depends on a subject's level of fitness and metabolic or gas exchange threshold. For these reasons and based on experience in our laboratory with this type of subject, we chose $120 \mathrm{~W}$ for the steady state exercise load to maximise the $\Delta \dot{\mathrm{V}}_{2}$ during exercise below the subject's $\dot{\mathrm{V}}_{2} \theta$, and we scrutinised each CWR test for evidence of upward drift. Five of the ten subjects whom we studied showed evidence of continuing upward drift of $\dot{\mathrm{V}}_{2}$ and were therefore excluded from further analysis. Although the reduction in the number of subjects was disappointing, we believe we were still able to show important correlations with the smaller number of subjects reported.

Other investigators have shown the time constant to be altered by cardiovascular and pulmonary disease. Koike et al ${ }^{41}$ showed a prolonged $\tau \dot{\mathrm{V}}_{2}$ in patients with a history of a myocardial infarction during submaximal constant rate exercise. When these patients were subdivided, those with a lower left ventricular ejection fraction $(<35 \%)$ manifested a significantly slower $\tau \dot{\mathrm{V}}_{2}$ than those with a higher ejection fraction $(\geqslant 35 \%)$. Yet there were no differences in $\dot{\mathrm{V}} \mathrm{O}_{2} \mathrm{MAX}$ or cardiac output at peak exercise during an IWR test. These investigators concluded that $\tau \dot{\mathrm{V}}_{2}$ during CWR exercise could be used as a sensitive and discriminate measure of impaired cardiac reserve. Casaburi et al ${ }^{42}$ showed evidence that physical training shortens $\tau \dot{\mathrm{V}}_{2}$ in patients with chronic obstructive pulmonary disease. After a structured exercise programme, the mean response time ( $\tau \dot{\mathrm{V}}_{2}$ plus a delay factor for phase I) decreased from 87 to 72 seconds $(p<0.001)$. Further studies evaluating $\tau \dot{\mathrm{V}}_{2}$ in other cardiopulmonary diseases are likely to substantiate its potential for clinical utility along with the other more commonly measured and investigated variables of aerobic fitness. However, we acknowledge that our inability to estimate a CWR that fell just below the metabolic threshold in some of our subjects is problematic and may significantly limit the clinical application of this approach.

The results of our study are consistent with the proposal that $\tau \dot{\mathrm{V}}_{2}$ is a valid variable of aerobic fitness in healthy subjects. Even in a small and fairly homogeneous group of normal subjects, we have shown that those who are less fit by virtue of lower $\dot{\mathrm{V}}_{2} \mathrm{MAX}$ and $\dot{\mathrm{V}}_{2} \theta$ have slower $\tau \dot{\mathrm{V}}_{2}$. Although we studied only five subjects, our protocol yielded $30 \mathrm{CWR}$ and 15 IWR tests for analysis. Furthermore, our subjects were homogeneous in terms of age, sex, and fitness level. Despite these factors, we were still able to show statistically significant and meaningful correlation between variables such as $\tau \dot{\mathrm{V}}_{2}$ and $\tau \dot{\mathrm{V}}_{2} \mathrm{MAX}$ or $\dot{\mathrm{V}}_{2} \theta$. Therefore, we conclude that $\tau \dot{\mathrm{V}}_{2}$ is a valid and reproducible variable of aerobic fitness. In addition, optimising the signal/noise ratio is key to counter the need for multiple repetitions. Lastly, $\tau \dot{\mathrm{V}}_{2}$ from an IWR test may have a different meaning from a CWR test. Currently, our testing methodology implies that only two repetitions are required if one obtains and averages both on$\tau \dot{\mathrm{V}}_{2}$ and off- $\tau \dot{\mathrm{V}} \mathrm{O}_{2}$ values. Future work interpreting both the on- $\tau \dot{\mathrm{V}}_{2}$ and off- $\tau \dot{\mathrm{V}}_{2}$ together may eliminate the need for more than one test. For research purposes in similar subjects, four tests are recommended when looking at either on- $\tau \dot{\mathrm{V}}_{2}$ or off- $\tau \dot{\mathrm{V}} \mathrm{O}_{2}$ separately. We have shown in this small group of normal subjects that on- $\tau \dot{\mathrm{V}}_{2}$ is highly correlated with off$\tau \dot{\mathrm{V}} \mathrm{O}_{2}$, which implies that the two time constants measure the same physiological entity. Furthermore, we have shown on- $\tau \dot{\mathrm{V}}_{2}$ to be inversely correlated with $\dot{\mathrm{V}}_{2} \mathrm{MAX}$ and $\dot{\mathrm{V}}_{2} \theta$ in a relatively homogeneous group of normal subjects. We hasten

Table 6 Ramp $\tau \dot{V}_{2}$ from incremental work rate tests in seconds

\begin{tabular}{llllllll}
\hline Subject & Ramp 1 & Ramp 2 & Ramp 3 & Mean & SD & CV (\%) & LOA (\%) \\
\hline 1 & 43.5 & 78.1 & 87.2 & 69.6 & 23.06 & 33 & 64.9 \\
2 & 43.4 & 49.3 & 45.8 & 46.2 & 2.97 & 6 & 12.5 \\
3 & 73.3 & 64.2 & 58.8 & 65.4 & 7.33 & 11 & 21.9 \\
4 & 60.2 & 41.5 & 54.4 & 52.0 & 9.57 & 18 & 36.1 \\
5 & 57.7 & 75.0 & 79.1 & 70.6 & 11.36 & 16 & 31.5 \\
Mean & 55.6 & 61.6 & 65.1 & 60.8 & & & \\
SD & 12.6 & 15.9 & 17.4 & & & &
\end{tabular}

$\mathrm{CV}$, Coefficient of variability; LOA, limits of agreement. 


\section{Take home message}

A reliable measure of $\tau \dot{V}_{2}$ can be obtained from a single CWR exercise protocol below the metabolic threshold for lactate accumulation by averaging the values obtained by monoexponential non-linear regression analysis of both the on- and off-transits. The arithmetic mean obtained in this manner from multiple transitions has been shown to be numerically similar to the $\tau \dot{\mathrm{V}} \mathrm{O}_{2}$ obtained by time alignment and monoexponential non-linear regression analysis of superimposed data.

to add that these conclusions do not necessarily apply to patients with cardiopulmonary disease who have a reduced amplitude of response below the gas exchange threshold. However, interestingly, in these patients, the confidence of determining $\tau \dot{\mathrm{V}}_{2}$ is somewhat improved by having more transient data because of the longer time constant.

\section{ACKNOWLEDGEMENTS}

We thank Stephanie Smooke, Paul Sedheva, and Jason Hove for their assistance in recruiting subjects, performing exercise tests, and data management.

\section{Authors' affiliations}

G H Markovitz, C B Cooper, Departments of Medicine and Physiology, UCLA School of Medicine, Los Angeles, CA 90095, USA

JW Sayre, Departments of Biostatistics and Radiological Sciences, UCLA School of Medicine

T W Storer, Exercise Science Laboratory, El Camino College, Torrance, CA 90506, USA

\section{REFERENCES}

1 Whipp BJ, Davis JA, Torres F, et al. A test to determine parameters of aerobic function during exercise. J Appl Physiol 1981;50:217-21.

2 Margaria R, Edwards HT, Dill DB. The possible mechanism of contracting and paying the oxygen debt and the role of lactic acid in muscular contraction. paying the oxygen debt and the rolec

3 Paterson DH, Whipp BJ. Aysmmetries of oxygen uptake transients at the onand offset of heavy exercise in humans. J Physiol (Lond) 1991;443:575-86.

4 Yoshida T, Whipp BJ. Dynamic asymmetries of cardiac output transients in response to muscular exercise in man. J Physiol (Lond) 1994:480:355-9.

5 Casaburi R, Barstow TJ, Robinson T, et al. Influence of work rate on ventilatory and gas exchange kinetics. J Appl'Physiol 1989;67:547-55.

6 Cooper CB, Lewis DA, Wasserman K. Geometric analysis of cumulative oxygen uptake to determine $\mathrm{VO}_{2}$ kinetics for constant work rate exercise [abstract]. Am Rev Respir Dis 1994; 145:A585.

7 Nery LE, Wasserman K, Andrews JD, et al. Ventilatory and gas exchange kinetics during exercise in chronic obstructive pulmonary disease. J Appl Physiol 1982;53:1594-602.

8 Sietsema KE, Cooper DM, Perloff JK, et al. Dynamics of oxygen uptake and heart rate responses during exercise in adults with cyanotic congenital heart disease. Circulation 1986;73:1137-44.

9 Barstow TJ, Casaburi R, Wasserman K. Oxygen uptake kinetics and the $\mathrm{O}_{2}$ deficit as related to exercise intensity and blood lactate. J Appl Physiol 1993;75:755-62.

10 Hughson RL, Inman MD. Oxygen uptake kinetics from ramp work tests: variability of single test values. J Appl Physiol 1986;61:373-6.

11 Phillips SM, Green HJ, MacDonald MJ, et al. Progressive effect of endurance

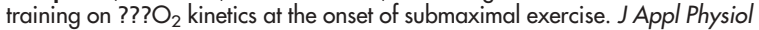
1995; 79:1914-20

12 Whipp BJ, Ward SA, Lamarra N, et al. Parameters of ventilatory and gas exchange dynamics during exercise. J Appl Physiol 1982;52:1506-13.

13 Davis JA, Whipp BJ, Lamarra N, et al. Effect of ramp slope on measurement of aerobic parameters from the ramp exercise test. Med Sci Sports Exerc 1982; 14:339-43

14 Buchfuhrer MJ, Hansen JE, Robinson TE, et al. Optimizing the exercise protocol for cardiopulmonary assessment. J Appl Physiol 1983;55:1558-64.

15 Karlsson H, Lindborg B, Linnarsson D. Time courses of pulmonary gas exchange and heart rate changes in supine exercise. Acta Physiol Scand 1975;95:329-40.

16 Gerbino A, Ward SA, Whipp BJ. Effects of prior exercise on pulmonary gas exchange kinetics during high-intensity exercise in humans. J Appl Physiol 1996:80:99-107.

17 Roston WI, Whipp BJ, Davis JA, et al. Oxygen uptake kinetics and lactate concentration during exercise in man. Am Rev Respir Dis 1987; 135:1080-4.
18 Zhang $\mathrm{YY}$, Wasserman $\mathrm{K}$, Sietsema K, et al. $\mathrm{O}_{2}$ uptake kinetics in response to exercise. Chest 1993;103:735-41.

19 Whipp BJ, Wasserman K. Oxygen uptake kinetics for various intensities of constant load work. J Appl Physiol 1972;33:351-6.

20 Beaver WL, Wasserman K, Whipp BJ. A new method for detecting anaerobic threshold by gas exchange. J Appl Physiol 1986;60:2020-7.

21 Sue DY, Wasserman K, Moricca RB, et al. Metabolic acidosis during exercise in patients with chronic obstructive pulmonary disease: use of the $\mathrm{V}$-slope method for anaerobic threshold detection. Chest 1988;94:931-8.

22 Wasserman K. Breathing during exercise. N Engl J Med 1978;298:780-5.

23 Snedecor GW, Cochrane WG. Statistical methods, 8th ed. Ames, IA: lowa State University Press, 1989

24 Atkinson G, Nevill AM. Statistical methods for assessing measurement error (reliability) in variables relevant to sports medicine. Sports Med 1998;4:217-38.

25 Consolazio CF, Johnson RE, Pecora U. Physiological measurements of metabolic functions in man. New York: McGraw Hill, 1963.

26 Jones NL, Makrides L, Hitchcock C, et al. Normal standards for an incremental progressive cycle ergometer test. Am Rev Respir Dis 1985;131:700-8.

27 American Thoracic Society. Standardization of spirometry. 1994 Update. Am J Respir Crit Care Med 1995; 152:1107-36.

28 Wasserman K, Whipp BJ. Exercise physiology in health and disease. Am Rev Respir Dis 1975;112:219-49.

29 Whipp BJ, Wasserman K. Efficiency of muscular work. J Appl Physiol 1969;26:644-8.

30 Shepherd RJ, Allen C, Benade AJS, et al. The maximum oxygen uptake. An international reference standard of cardiorespiratory fitness. Bull WHO 1968:38:757-64.

31 Sietsema KE, Daly JA, Wasserman K. Early dynamics of $\mathrm{O}_{2}$ uptake and heart rate as affected by exercise work rate. J Appl Physiol 1989;67:2535-41.

32 Chilibeck PD, Paterson DH, Petrella RJ, et al. The influence of age and cardiorespiratory fitness on kinetics of oxygen uptake. Can J Appl Physiol 1996:21:185-96.

33 Griffiths TL, Henson LC, Whipp BJ. Influence of inspired oxygen concentration on the dynamics of the exercise hyperpnoea in man. J Physiol (Lond) 1986:380:387-403

34 Ozyener F, Rossiter HB, Ward SA, et al. Influence of exercise intensity on the on- and off-transient kinetics of pulmonary oxygen uptake in humans. J Physiol (Lond) 2001;533:891-902.

35 Lamarra N, Whipp BJ, Ward SA, et al. Effect of interbreath fluctuations on characterizing exercise gas exchange kinetics. J Appl Physiol 1987;62:2003-12.

36 Knox AJ, Morrison JFJ, Muers MR. Reproducibility of walking tests in chronic obstructive airways disease. Thorax 1988;43:388-92.

37 Powers SK, Dodd S, Beadle RE. Oxygen uptake kinetics in trained athletes differing in $\mathrm{VO}_{2}$ max. Eur J Appl Physiol 1985;54:306-8.

38 Wasserman K. Coupling of external and internal respiration. Am Rev Respir Dis 1984;129(suppl):S21-4.

39 Camus G, Atchou G, Bruckner JC, et al. Slow upward drift of $\mathrm{VO}_{2}$ during constant-load cycling in untrained subjects. Eur J Appl Physiol 1988;58:197-202.

40 Whipp BJ, Mahler M. Dynamics of pulmonary gas exchange during exercise. In: West JB, eds. Pulmonary gas exchange. New York: New York Academic Press, 1980;2:33-96

41 Koike A, Hiroe M, Adachi H, et al. Oxygen uptake kinetics are determined by cardiac function at onset of exercise rather than peak exercise in patients with prior myocardial infarction. Circulation 1994;90:2324-32.

42 Casaburi R, Porszasz J, Burns MR, et al. Physiologic benefits of exercise training in rehabilitation of patients with severe chronic obstructive pulmonary disease. Am J Respir Crit Care Med 1987; 155:1541-51.

\section{COMMENTARY}

The ability to quantify the time course of phase II $\mathrm{O}_{2}$ kinetics provides us with a tool by which aerobic responses to training and disease can be assessed without participants exercising to volitional exhaustion. This paper presents a method that potentially reduces the stress on the participant while at the same time not compromising the level of validity of the test. The use of the on and off transit $\mathrm{VO}_{2}$ data from a constant work rate test is promising, but does not address the problem of the need to still identify the metabolic threshold, which, as the authors point out, if not correctly determined could lead to $\mathrm{VO}_{2}$ drift and the development of the slow component. This paper shows that a consensus needs to be drawn as to the appropriate method for the assessment of the time course of phase II $\mathrm{O}_{2}$ kinetics.

D A Gordon Anglia Polytechnic University, Sport Science/School of Applied Sciences, David Building, East Road, Cambridge CB1 1PT, UK; d.a.gordon@anglia.ac.uk 